\title{
Comparación de la efectividad del midazolam en niños: via oral y via intranasal
}

Castro ES, Díaz-Pizán ME, Valdivieso M. Comparación de la efectividad del midazolam en niños : via oral y via intranasal. Rev Estomatol Herediana 2005; 15 (2) : 133 - 138.

\section{Esmeralda Soledad Castro Jerí1 Maria Elena Díaz-Pizán² Mónica Valdivieso Vargas Machuca²}

${ }^{1}$ Cirujano Dentista

2 Docente del Departamento Académico de Estomatología del Niño y del Adolescente.

Facultad de Estomatología. Universidad Peruana Cayetano Heredia.

\section{RESUMEN}

El propósito del presente estudio fue evaluar la efectividad de dos formas de administración de midazolam, vía oral e intranasal en la modificación de la conducta de niños en edad preescolar quienes recibieron tratamiento dental. Participaron 20 niños con edades entre los 22 a 68 meses, quienes recibieron midazolam en dosis de $0,5 \mathrm{mg} / \mathrm{kg}$ de peso administrado por vía oral y $0,2 \mathrm{mg} / \mathrm{kg}$ de peso administrado por vía intranasal. A todos los niños se les registró la presión arterial, frecuencia cardiaca y saturación de oxigeno antes, durante y después de la sedación. Se evaluaron los cambios de conducta utilizando la escala de Houpt. Los rangos alcanzados en los parámetros llanto, somnolencia y movimientos no difieren significativamente en ambas vías. Los signos vitales se mantuvieron estables en los tres momentos evaluados para ambas vías (antes, durante y después de la sedación). No se presentaron reacciones adversas en ninguno de los casos. El tiempo de recuperación promedio para el grupo sedado por vía oral fue de 108,1 min. y para el grupo de niños sedados por vía intranasal fue de 94,6 minutos. Este estudio demostró la eficacia del midazolam administrado tanto por vía oral como por vía intranasal en el tratamiento dental bajo sedación consciente en niños en edad preescolar, destacando la ventaja de la vía intranasal en el tiempo de recuperación del paciente ya que éste fue menor.

Palabras clave: MIDAZOLAM. administración \& dosificación / ODONTOLOGÍA PEDIÁTRICA
Effectivemness of oral and intranasal Midozolam in pediatric dentistry ABSTRACT

The purpose of this study is to assess the effectiveness of the two forms of administration of midazolam, oral and intranasal, in the modification of pre-school and school-age children's behavior under dental treatment. 20 children participated, ages, 22 to 68 months; were administered midazolam in doses of $0.5 \mathrm{mg} / \mathrm{kg}$ of weight orally or $0.2 \mathrm{mg} / \mathrm{kg}$ intranasal. All the subjects were checked for blood pressure, cardiac frequency and oxygen saturation prior, during and after sedation. Changes in behavior were evaluated using Houpt scale. The ranges obtained in the parameters cry, somnolence and movement did not differ significantly in either administration method. Vital signs remained stable at the three moments measured for both ways of administration (before, during and after sedation). No adverse reactions were observed in any of the cases. The mean recovery time for the group sedated orally was 108.1 min and for the group of children sedated intranasally, $94.6 \mathrm{~min}$. This study demonstrated the efficacy of Midazolam administered bith orally and intranasally for dental treatment under conscious sedation in pre-school age children. The results favor the intranasal way due to the shortest recovery time.
Av. Angelica Gamarra 1495 Lima - 39, Perú Teléfono: 531-6057

e-mail: esmeralda_castroj@ hotmail.com
Keywords: MIDAZOLAM. administration \& dosage / PEDIATRIC DENTISTRY

\section{Introducción}

El midazolam es uno de los medicamentos más utilizados para sedación en odontología pediátrica siendo las vías oral e intranasal las que tienen mayor aceptación, por la facilidad de su administración.

Diversos estudios demuestran la eficacia de la vía oral en el tratamiento dental de niños pequeños. En 1996, Reeves et al.(1) compararon la efectividad del midazolam por vía oral en dosis de 0,5 $\mathrm{mg} / \mathrm{kg}$ administrado con acetaminofeno frente al hidrato de cloral con hidroxicina en el tratamiento odontológico de 40 niños comprendidos entre 27 y 73 meses de edad. Sus resultados demostraron que el midazolam por vía oral es un agente sedante efectivo para procedimientos dentales cortos, aunque no se observaron mayores ventajas en los cambios de conducta logrados de un grupo a otro. En 1999, Fraone et al.(2) evaluaron la conducta de 61 niños, con edades comprendidas entre los 24 y 58 meses de edad, tratados bajo sedación consciente. Encontrando que, el midazolam administrado por vía oral en dosis de $0,5 \mathrm{mg} / \mathrm{kg}$ de peso corporal, mejoraba la conducta en estos niños ansiosos frente al tratamiento dental. No existieron diferencias significativas en la conducta al comparar tres grupos de diferentes edades, así como tampoco cambios significativos en su fisiología.

En 2002, Singh et al.(3) realizaron una comparación de la eficacia entre el midazolam administrado por vía oral y otros dos agentes sedativos, el triclofos y la prometazina para el tratamiento de niños con edades entre 3 y 9 años de edad. Encontrando al midazolam como una buena elección para la sedación consciente. Igualmente, Wilson et al.(4) (2002) encontraron al midazolam administrado por vía oral como una buena elección para las extracciones dentales en pacientes con edades entre los 10 y 16 años, comparándolo con el óxido nitroso.

Karl et al.(5) (1993) compararon la aceptación y eficacia del midazolam administrado por vía intranasal y por vía submucosa en 93 niños entre los 6 meses y 10 años de edad y, a pesar de observar la misma eficacia por ambas vías, la administración por vía intranasal fue la menos aceptada por la sensación de "quemazón" que produce al primer contacto con la mucosa nasal; la cual luego de unos segundos desaparece, provocando molestias sólo al inicio de su aplicación. En 1993, Fukuta et al.(6) evaluaron el efecto conseguido al sedar a pacientes física y/o mentalmente impedidos con edades comprendidas entre los 2 a 21 años, con una dosis de $0,2 \mathrm{mg} / \mathrm{kg}$ de midazolam intranasal, obteniendo como resultados un rápido inicio de la sedación, cambios positivos de la conducta y ausencia de efectos adversos de consideración. En 2001, Al-Rakaf et al.(7) compararon tres diferentes dosis de midazolam intranasal, evaluando la efectividad de la sedación con $0,3 \mathrm{mg} /$ $\mathrm{kg}, 0,4 \mathrm{mg} / \mathrm{kg}$ y $0,5 \mathrm{mg} / \mathrm{kg}$ de peso cor- 
poral en 38 niños ansiosos de 2 a 5 años de edad, llegando a la conclusión que las tres dosis de midazolam fueron igualmente efectiva en la modificación del comportamiento del niño no cooperador. Asimismo encontraron entre sus ventajas un inicio rápido de sedación y que no era necesario que el paciente acudiera en su cita en ayunas; a diferencia de la sedación por vía oral, en la que acudir en ayunas, es indispensable.

Entre los estudios que comparan la eficacia de ambas técnicas encontramos a Hartgraves et al.(8) (1994) quienes compararon la administración de midazolam por vía intranasal en dosis de $0,2 \mathrm{mg} / \mathrm{kg}$ $v s$ midazolam administrado por vía oral en dosis de $0,5 \mathrm{mg} / \mathrm{kg}$; estableciendo que ambas vías son de utilidad en el tratamiento odontológico de niños preescolares altamente ansiosos.

La escala utilizada en este trabajo para la comparación está basada en un estudio que realizaron Houpt et al.(9) en 1993, en donde se contó con la participación de 25 odontopediatras; quienes evaluaron el uso de fármacos más frecuentes utilizados en sedación consciente en odontología pediátrica, registrándose los rangos de conducta y clasificándolos en diferentes escalas para las variables llanto, somnolencia y movimiento. Además, se utilizó una escala de conducta que abarcaba todo el tratamiento en general. Esta escala ha sido utilizada posteriormente por otros investigadores para comparar la efectividad de diversos fármacos (10). En este estudio la comparación entre ambas vías de administración se hace en base a esta escala.

El propósito de la presente investigación fue comparar la efectividad del midazolam administrado por vía intranasal y por vía oral como agente sedante en pacientes en edad preescolar cuyo comportamiento impedía realizar el tratamiento dental. Ambas vías de administración, oral e intranasal, pueden ser consideradas como convenientes y efectivas para el tratamiento odontológico en niños de edad preescolar con conducta "no cooperadora".

\section{Materiales y método}

Este estudio fue realizado en el Servicio de Postgrado en Odontopediatria en la Clínica Dental de la Universidad Peruana Cayetano Heredia en Lima, Perú. Participaron 20 niños, entre los 22 y 68 meses de edad, con conducta defi-

Tabla 1. Escala de conducta desarrollada por Houpt.

\begin{tabular}{|c|c|c|c|}
\hline escala & llanto & somnolencia & movimiento \\
\hline 1 & histérico & despierto, forcejeando & $\begin{array}{l}\text { movimientos violentos } \\
\text { interrumpiendo el tratamiento }\end{array}$ \\
\hline 2 & continuo, fuerte & despierto, quieto & $\begin{array}{l}\text { movimientos fuertes que } \\
\text { dificultan el tratamiento }\end{array}$ \\
\hline 3 & $\begin{array}{l}\text { intermitente, } \\
\text { moderado }\end{array}$ & somnoliento & $\begin{array}{l}\text { ligeros movimientos que no } \\
\text { interfieren con el tratamiento }\end{array}$ \\
\hline 4 & no hay llanto & dormido & no hay movimiento \\
\hline
\end{tabular}

nitivamente negativa según la escala de Frankl (11) distribuidos en dos grupos: Grupo A, para la aplicación de midazolam por vía intranasal, integrado por diez pacientes, con promedio de edad de 34,7 meses. Grupo B, para la aplicación de midazolam por vía oral integrado por diez pacientes, con promedio de edad de 41,1 meses.

Los pacientes fueron examinados previamente por un médico pediatra quien determinó su condición médica saludable para la sedación.

Los padres de los niños fueron informados del tipo de tratamiento, ventajas y desventajas de la sedación y recibieron instrucciones escritas precisas sobre el procedimiento de sedación; y se obtuvo el permiso del padre para que su hijo forme parte del presente estudio mediante un consentimiento informado validado.

Todos los procedimientos clínicos fueron programados para ser realizados en citas a primeras horas de la mañana, siendo obligatorio que el paciente acuda en ayunas. El operador se aseguró que el paciente no presentara ningún tipo de resfrío o secreción nasal. Los tratamientos fueron realizados por un residente del postgrado del Área de Odontología Pediátrica, previamente calibrado por los docentes del área, y con ayuda de dos asistentes dentales debidamente entrenados en tratamientos bajo sedación consciente. El día de la cita, los pacientes fueron asignados por el operador en uno de los dos grupos: ya sea el de vía oral o intranasal, luego de lo cual se determinó la dosis para cada niño según su peso. El investigador registró en la ficha clínica la conducta observada según la escala de Houpt (Tabla 1); los signos vitales y las reacciones adversas.

Los pacientes del Grupo A recibieron midazolam en dosis de $0,2 \mathrm{mg} / \mathrm{kg}$ de peso corporal por vía intranasal, la cual se llevó a cabo por instilación en una fosa nasal mediante goteo, con una jeringa de tuberculina de $1 \mathrm{ml}$. El tiempo de espera para el efecto de la droga fue de 10 minutos, luego de lo cual se dió comienzo al tratamiento odontológico (Fig. 1).

Los pacientes del Grupo B recibieron midazolam en dosis de $0,5 \mathrm{mg} / \mathrm{kg}$ de peso corporal por vía oral. El fármaco en tabletas fue dividido según la dosis calculada disolviéndose posteriormente en un vaso un jugo de frutas, a fin de no ser rechazado por el niño ya que su sabor es bastante desagradable. El tiempo de espera para el inicio de los efectos sedantes fue de 30 minutos, luego de lo cual se dio inicio al tratamiento (Fig. 2).

Durante el tratamiento, todos los pacientes fueron inmovilizados con restricción física, utilizándose para ello un papoose board (Olympic Papoose Board, Olympic Medical Corp., Seattle, WA) en el sillón dental, con el objetivo de minimizar cualquier posible injuria como resultado de algún movimiento abrupto. Así mismo, todos los niños fueron sometidos a un permanente y minucioso control de los parámetros hemodinámicos y respiratorios mediante monitorización. Se registró: presión arterial, frecuencia cardiaca y saturación de oxígeno antes de administrar el agente sedante, durante la sedación y después de terminado el tratamiento odontológico. La saturación de oxígeno fue continuamente monitoreada por medio de un oxímetro de pulso BCIÒ International 3301. La presión arterial y la frecuencia cardiaca fueron registradas con un tensiómetro de pulso digital CITIZENÒ, que fue colocado en la muñeca del brazo derecho de cada niño. El investigador fue el responsable de monitorizar y registrar los signos vitales del paciente. Terminado el tratamiento, el niño quedó al cuidado de la madre. Se realizó un seguimiento del niño por medio telefónico durante la noche, después de la sedación. 
El tiempo de recuperación estuvo basado en la estabilidad de sus funciones vitales y del retorno a la normalidad, particular atención se le dió a la reacción de respuesta del paciente a la llamada por su nombre, la reacción a la intervención de una tercera persona, por ejemplo: tocarle la mano, la capacidad para caminar y la ausencia de reacciones adversas.

\section{Resultados}

Conducta

La conducta fue evaluada utilizan- do la escala de Houpt et al.(9) Los parámetros tomados en cuenta fueron: llanto, somnolencia y movimiento, con rangos del 1 al 4. Los datos fueron analizados para determinar diferencias entre las dos vías de administración. Al realizar comparaciones estadísticas utilizando la prueba de U Mann-Whitney con $95 \%$ de significancia, se observó que los rangos alcanzados en los parámetros llanto y somnolencia no difieren significativamente en ninguna de sus fases $(p>0,05)$. Iguales resultados se obtuvieron al comparar el movimiento entre ambos grupos, excepto para las fases de DG (colocación del dique de goma) e IP (inicio del procedimiento clínico) en que se observaron diferencias estadísticamente significativas $(\mathrm{p}<0,05)$ siendo más favorables los rangos alcanzados en la vía oral (Fig. 3, 4, 5).

\section{Signos vitales}

La presión arterial, frecuencia cardiaca y saturación de oxígeno no cambiaron significativamente en ningu-

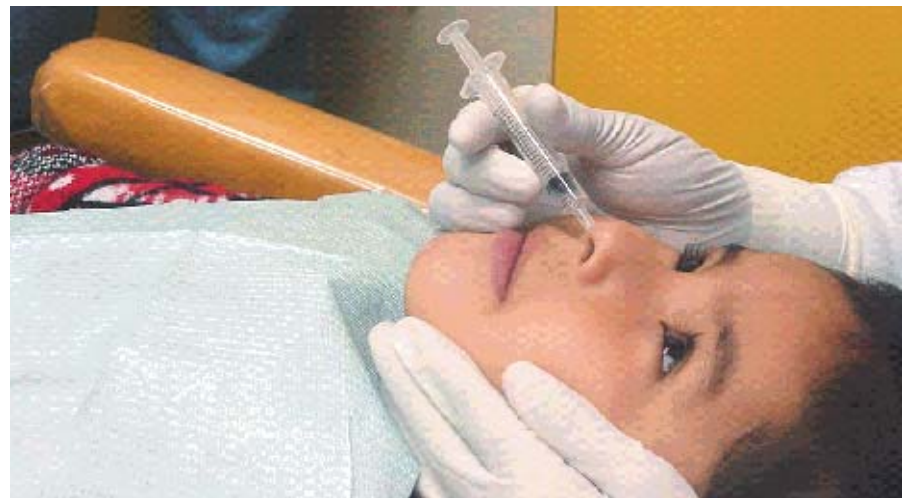

Fig. 1. Administración por vía intranasal

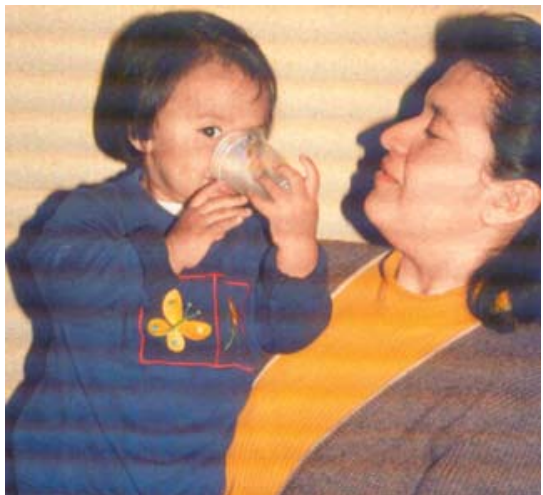

Fig. 2. Administración por vía oral

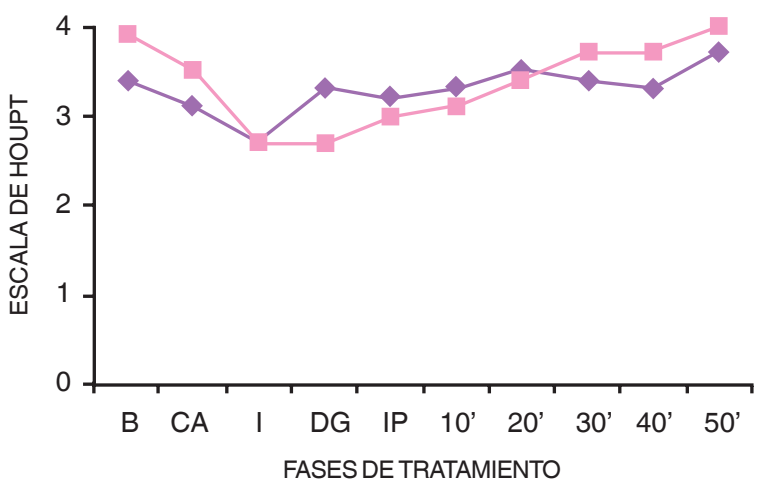

Fig. 3. Comparación de movimiento según vía de administración durante las diferentes fases de tratamiento. administración durante las diferentes fases de tratamiento.

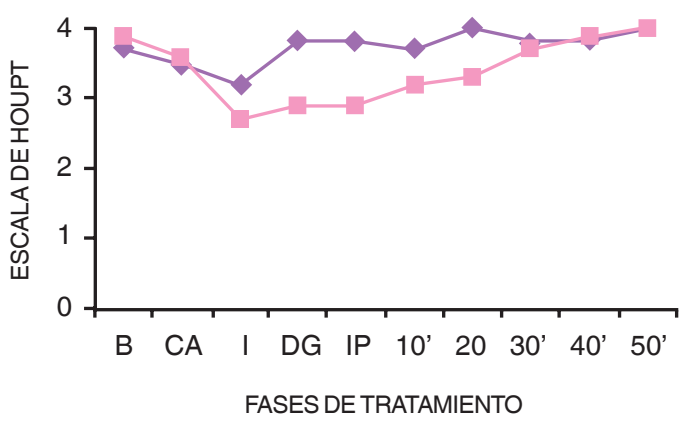

Fig. 5. Comparación de llanto según vía de administración durante las diferentes fases de tratamiento.

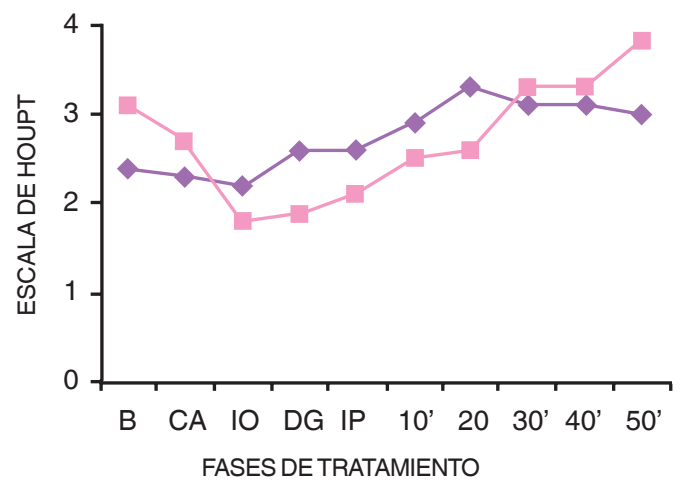

Fig. 4. Comparación de somnolencia según vía de

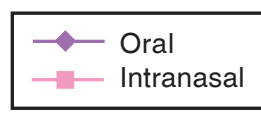

Leyenda:

B: Basal

CA: Colocación de Anestesia

I: Inicio del Procedimiento Clínico

DG: Colocación del Dique de Goma

IP: Inicio del tratamiento

10': A los 10 minutos de haber iniciado el tratamiento

20': A los 20 minutos de haber iniciado el tratamiento

$30^{\prime}$ : A los 30 minutos de haber iniciado el tratamiento

40': A los 40 minutos de haber iniciado el tratamiento

50': A los 50 minutos de haber iniciado el tratamiento 
no de los grupos, al compararlos en tres momentos diferentes: antes, durante (a los $10 \mathrm{~min}$. en el grupo A y $30 \mathrm{~min}$. en el grupo B) y después del tratamiento odontológico.

\section{Efectos adversos}

En ningún caso se dieron reacciones adversas, tales como: vómitos, vértigos, alucinaciones o daño tisular en la mucosa nasal o en la orofaringe; ni durante la sedación ni después de ella.

\section{Tiempo de recuperación}

En el grupo de midazolam por vía intranasal $(0,2 \mathrm{mg} / \mathrm{kg})$ el tiempo de recuperación más corto fue de 80 minutos y el más extenso de 110 minutos. Mientras que para el grupo que se administró midazolam por vía oral $(0,5 \mathrm{mg} / \mathrm{kg}) \mathrm{el}$ tiempo más corto fue de 95 minutos y el más largo 120 minutos. Observándose un promedio de tiempo de recuperación para el grupo A (vía intranasal) de 94,6 minutos y para el grupo B (vía oral) de 108, 1 minutos. La prueba t-student determinó diferencias estadísticamente significativas $(\mathrm{p}<0,05)$ en cuanto al tiempo de recuperación de ambos grupos (Tabla 2).

\section{Discusión}

Los resultados muestran que ambas vías de administración son similarmente efectivas. Confirmando los hallazgos de

Tabla 2. Comparación del tiempo de recuperación en ambos grupos

\begin{tabular}{lcccc}
\hline \multicolumn{5}{c}{$\begin{array}{l}\text { tiempo de recuperación } \\
\text { (en minutos) }\end{array}$} \\
\hline vía & mín & $\max$ & $\mathrm{X}$ & $\mathrm{DE}$ \\
oral & 90 & 120 & 108,1 & 7,78 \\
intranasal & 80 & 110 & 94,6 & 7,99 \\
\hline
\end{tabular}

Prueba t-student $(\mathrm{p}<0,05)$

Hartgraves y Primosch (8) quienes al comparar la efectividad de la administración del midazolam por vía intranasal en dosis de $0,2 \mathrm{mg} / \mathrm{kg}$ con la vía oral en dosis de $0,5 \mathrm{mg} / \mathrm{kg}$ en 100 niños de $1,5 \mathrm{a}$ 6 años de edad, concluyeron que ambas vías son seguras y efectivas en ayudar a modificar la conducta de pacientes odontopediátricos no cooperadores durante el tratamiento odontológico.

Las dosis utilizadas de $0,2 \mathrm{mg} / \mathrm{kg}$ y $0,5 \mathrm{mg} / \mathrm{kg}$ en la administración por vía intranasal y oral respectivamente, estuvieron basadas en resultados positivos registrados en estudios previos, respecto a la efectividad del midazolam como sedante y dosis bajas utilizadas en el tratamiento odontológico de pacientes pediátricos $(1,2,6,8-10,12,14-19)$.

La evaluación de la conducta bajo los efectos del sedante se realizó utilizando la escala de clasificación de conducta diseñada por Houpt et al.(9) Esta evalúa la conducta en base a tres parámetros: llanto, somnolencia y movimiento, encontrándose que no hubieron diferencias significativas con llanto y somnolencia; sin embargo, en lo referente al movimiento sí hubieron diferencias significativas en las fases DG (colocación del dique de goma) e IP (inicio de procedimiento clínico) observándose menos movimientos en los niños sedados por vía oral. Esto puede deberse a que el grupo que recibió el midazolam por vía intranasal podría haber alcanzado una mayor concentración del agente sedante en el plasma en un mayor tiempo que el que se esperó (más de 10 minutos). Razón por la que en las siguientes fases del acto operatorio se observan valores similares para ambos grupos.

Reeves et al.(1) en 1996, al comparar dos combinaciones de agentes sedantes en 40 niños con edades comprendidas entre los 27 y 43 meses, no hallaron diferencias significativas con relación a llanto y movimiento, pero con el parámetro somnolencia sí se observaron diferencias estadísticamente significas durante la inyección del anestésico local, siendo los resultados más favorables para el grupo sedado con hidrato de cloral/hidroxicina, que los observados en el grupo que recibió midazolam/ acetaminofeno.

Las observaciones clínicas realizadas en el presente estudio, confirman los resultados hallados en investigaciones reportadas en la literatura odontopediátrica. Así, pudimos observar que por vía intranasal el efecto sedante se dio a los 10 minutos aproximadamente de haberse administrado la dosis. Estos datos son similares a los observados por Wilton et al.(12) (1988) quienes administraron midazolam por vía intranasal como premedicación antes de la anestesia general, en dosis de $0,2 \mathrm{mg} / \mathrm{kg}$, a niños de corta edad y observaron el inicio de sus efectos a los 10 minutos de haber sido administrado.

Por otro lado, Al Rakaf et al.(7) (2001) observaron que el inicio de los efectos de la sedación se da a los 10 minutos de haber sido administrado el fármaco, independientemente de la dosis administrada $(0,3 ; 0,4$ ó $0,5 \mathrm{mg} / \mathrm{kg})$. Esto es comparable a estudios previos en los que se observa el pico de concentración plasmática a los 10 minutos (13).

En cuanto al comienzo de los efectos sedantes, para los que recibieron el midazolam por vía oral, se dieron a los 30 minutos de su administración. Coincidiendo con ello, Feld et al.(16) (1990) en su estudio del midazolam administrado por vía oral en 124 niños de 1 a 10 años de edad, observaron que a los 30 minutos de haber sido administradas las dosis de 0,5 y $0,75 \mathrm{mg} / \mathrm{kg}$ respectivamente, se comenzaban a apreciar los efectos del sedante; sin notar ventajas en la dosis mayor.

Una desventaja que presenta la sedación intranasal es el hecho de no ser bien aceptada por el paciente debido a la sensación de "quemazón" que este siente al momento de la instilación, razón por la que se requiere de restricción física para su administración. ${ }^{20}$ Con ello concuerdan Shapira et al.(10) (1996) quienes observaron en su estudio de 58 niños entre 2 y 4 años de edad, que el midazolam por vía intranasal causaba quemazón transitoria y no podía ser adecuadamente utilizado en niños que acudían a la cita con algún tipo de secreción nasal.

Otra desventaja es que se puede producir expulsión parcial de la dosis, debido a un considerable volumen de la solución aplicada o por una mala técnica en la administración de esta $(6,7,17,21)$. Es por ello que algunos sugieren no trabajar con dosis mayores a la utilizada en este estudio $(0,2 \mathrm{mg} / \mathrm{kg})$ cuando se administra por vía intranasal, puesto que mayores dosis requieren de mayor volumen de solución, lo cual podría resultar en más riesgos de estornudos o tos con probable expul- 
sión de parte de la dosis $(6,17,21)$.

En la administración por vía oral se pudo observar una mejor aceptación del paciente. Esto se debió a que el medicamento fue mezclado con un jugo de sabor agradable para el niño, lo cual trataba de contrarrestar el mal sabor del fármaco. En estudios previos, Hartgraves (8) (1994) contrarrestó el mal sabor mezclando el midazolam con $25 \mathrm{mg}$ de hidroxicina azucarada, con lo cual logró la aceptación del paciente. Igualmente, Feld et al.(16) (1990) usan jugo de manzana para darle mejor sabor y lograr la aceptación de pacientes al administrárseles el midazolam por vía oral.

El ayuno previo es otro problema asociado al uso del midazolam administrado por vía oral. Los niños acuden a su cita con hambre y es probable que esto influya en su comportamiento, razón por la que investigadores como $\mathrm{Al}$ Rakaf et al.(7) (2001) realizaron un estudio comparando la efectividad de tres dosis diferentes $(0,3 ; 0,4$ y $0,5 \mathrm{mg} / \mathrm{kg})$ administradas por vía intranasal en niños que acudieron a su cita en ayunas $y$ en no ayunas, pudiendo observar que la efectividad de la administración por esta vía no requirió de ayuno previo.

En cuanto a los signos vitales, no hubieron diferencias estadísticamente significativas en la presión arterial, frecuencia cardiaca y saturación de oxígeno durante toda la evaluación del estudio, tanto en los pacientes sedados por vía oral como en los que se administró la droga por vía intranasal. La presión arterial sistólica tendió a aumentar y luego volvió a normalizarse sin cambios significativos y dentro de los límites normales tanto en el grupo de administración por vía oral como vía intranasal.

Al valorar la saturación de oxígeno, observamos que en algunos casos este descendió a niveles inferiores al 95\% durante breves segundos, lo cual luego de realizar la hiperextensión del cuello del niño o aflojarse la restricción física a nivel del tórax se logró ventilación adecuada y por lo tanto, el retorno a los valores normales.

Nuestros hallazgos respecto a la estabilidad de los signos vitales confirman las conclusiones de estudios anteriores como el de Al Rakaf(7) (2001), en el que se muestra que el midazolam es seguro y efectivo para procedimientos odontológicos bajo sedación consciente.

En el estudio de Fukuta et al.(22) (1994), estos signos vitales tendieron a incrementarse luego de 10 a 20 minutos de haber sido administrado el sedante por vía intranasal en pacientes adultos de 19 a 25 años de edad, tendencia que fue descendiendo hasta alcanzar nuevamente los niveles iniciales. Es probable que esta tendencia de incremento al inicio sea resultado del stress y/o dolor provocado por la administración intranasal.

No hubieron reportes de náuseas, vómitos u otros efectos adversos ni durante ni después de haber concluido la sedación. Lo que nos confirma la seguridad de las dosis utilizadas en las vías oral e intranasal $(2,6,17,23)$.

El tiempo de recuperación del grupo A (vía intranasal) se caracterizó porque los efectos sedativos fueron desapareciendo con mayor rapidez, teniendo un promedio de 94,6 minutos. Mientras que el grupo B (vía oral) tuvo un promedio de tiempo de recuperación fue de 108,1 minutos. Esto proporciona mayor ventaja a la vía intranasal cuando las necesidades del tratamiento dental del paciente implica procedimientos cortos como una exodoncia simple o algún tratamiento de emergencia. Pero de requerirse tratamientos más extensos como tratamientos pulpares u operatorias es preferible el uso de la vía oral.

Fukuta et al.(22)(1994), al administrar el midazolam por vía intranasal observaron un promedio de 129,3 minutos para el tiempo de recuperación al utilizar la dosis de $0,2 \mathrm{mg} / \mathrm{kg}$ y de $131,8 \mathrm{mi}-$ nutos para la dosis de $0.3 \mathrm{mg} / \mathrm{kg}$ en 43 pacientes de 5 a 20 años de edad mentalmente discapacitados. Posteriormente, en 1997, estos mismos autores (17) observaron que todos los participantes de su estudio (quince jóvenes de 19 a 25 años de edad) mostraron sedación satisfactoria entre los primeros 15 a 20 minutos hasta los 55 a 60 minutos post administración del medicamento. En tanto los pacientes sedados con dosis de 0,2 como los sedados con $0,3 \mathrm{mg} / \mathrm{kg}$ recuperaron con normalidad sus funcio- nes corporales entre los 150 y 180 minutos luego de la administración del sedante. En el presente estudio se observó un tiempo de recuperación menor que el observado por Fukuta et al.(17) (1997) debido a que ellos emplearon midazolam combinado con óxido nitroso, técnica mediante la cual se puede prolongar el tiempo de sedación.

La vía de administración más utilizada en nuestro medio es la oral, debido a su facilidad de administración. Sin embargo, creemos que también debería considerarse la administración por vía intranasal como una alternativa efectiva y segura y sobretodo por las ventajas que ofrece tales como: menor tiempo de recuperación y no requerir de ayuno previo. Es de sugerir de nuestra parte realizar más estudios comparativos venciendo ciertas limitaciones que nosotros encontramos, tales como la poca aceptación de los odontopediatras para querer sedar por vía intranasal, razón por la cual tuvimos un numero reducido de participantes para la muestra de este estudio.

\section{Conclusiones}

- Los resultados obtenidos muestran que ambas vías de administración (oral e intranasal) son igualmente efectivas en cuanto a la modificación de la conducta según los parámetros evaluados: llanto, sueño y movimiento. Sólo en dos fases operatorias la vía oral mostró puntajes más altos al evaluar el movimiento (menos movimientos del niño); mostrando diferencias estadísticamente significativas.

- Ambas vías de administración son seguras pues los cambios fisiológicos observados están dentro de los límites normales sin mostrar diferencias estadísticamente significativas. - Ninguna de las dos vías de administración del midazolam presentaron algún tipo de reacciones adversas en el presente trabajo de investigación.

- El tiempo promedio de recuperación de la sedación por vía intranasal fue de 94,6 minutos mientras que por vía oral fue de 108,1, mostrándose diferencias estadísticamente significati- 
vas entre ambos grupos.

- Ambas vías de administración, oral e intranasal, pueden ser consideradas como convenientes y efectivas para el tratamiento odontológico de niños preescolares con conducta "no cooperadora". Sin embargo, la vía intranasal puede ser más ventajosa por su rápido comienzo del efecto sedante y su menor tiempo de recuperación.

\section{Referencias bibliográficas}

1. Reeves ST, Wiedenfeld KR, Wrobleski J, Hardin CL, Pinosky ML. A randomized double-blind trial of chloral hydrate/hydroxyzine versus midazolam/acetaminophen in the sedation of pediatric dental outpatients. ASDC J Dent Child 1996; 63(2):95-100.

2. Fraone $\mathrm{G}$, Wilson $\mathrm{S}$, Casamassimo PS, Weaver J 2nd, Pulido AM. The effect of orally administered midazolam on children of three age groups during restorative dental care. Pediatr Dent 1999; 21(4):235-41.

3. Singh N, Pandey RK, Saksena AK, Jaiswal JN. A comparative evaluation of oral midazolam with other sedatives as premedication in pediatric dentistry. J Clin Pediatr Dent 2002; 26(2):161-4.

4. Wilson S, Easton J, Lamb K, Orchardson R, Casamassimo P. A retrospective study of chloral hydrate, meperidine, hidroxyzine, and midazolam regimens used to sedated children for dental care. Pediatr Dent 2000; 22(2):107-112.

5. Karl HW, Rosenberger JL, Larach MG, Ruffle JM. Transmucosal administration of midazolam for premedication of pediatric patients. Comparison of the nasal and sublingual routes. Anesthesiology 1993; 78(5):885-91.

6. Fukuta O, Braham RL, Yanase H, Atsumi N, Kurosu K. The sedative effect of intranasal midazolam administration in the dental treatment of patients with mental disabilities. Part 1. The effect of a $0.2 \mathrm{mg} / \mathrm{kg}$ dose. J Clin Pediatr Dent 1993; 17(4):231-7.

7. al-RakafH, Bello LL, Turkustani A, Adenubi JO. Intra-nasal midazolam in conscious sedation of young paediatric dental patients. Int $\mathrm{J}$ Paediatr Dent 2001; 11(1):33-40.

8. Hartgraves PM, Primosch RE. An evaluation of oral and nasal midazolam for pediatric dental sedation. ASDC J Dent Child 1994; 61(3):175-81.

9. Houpt MI. Project USAP_-Part III: Practice by heavy users of sedation in pediatric dentistry. ASDC J Dent Child 1993; 60(3):183-5.

10. Shapira J, Holan G, Botzer E, Kupietzky A, Tal E, Fuks AB. The effectiveness of midazolam and hydroxyzine as sedative agents for young pediatric dental patients. ASDC J Dent Child. 1996 NovDec;63(6):421-5.

11. Frankl S, Shiere F, Fogels H. Should the parent remain with the child in the dental operatory? ASDC J Dent Child 1962; 29:150-163.

12. Wilton NC, Leigh J, Rosen DR, Pandit UA. Preanesthetic sedation of preschool children using intranasal midazolam. Anesthesiology 1988; 69(6):972-5.

13. Karl HW, Keifer AT, Rosenberger JL, Larach MG, Ruffle JM. Comparison of the safety and efficacy of intranasal midazolam or sufentanil for preinduction of anesthesia in pediatric patients. Anesthesiology 1992; 76(2):209-15.

14. Twersky RS, Hartung J, Berger BJ, McClain J, Beaton C. Midazolam enhances anterograde but not retrograde amnesia in pediatric patients. Anesthesiology 1993; 78(1):51-5.

15. Wilson S, Easton J, Lamb K,
Orchardson R, Casamassimo P. A retrospective study of chloral hydrate, meperidine, hydroxyzine, and midazolam regimens used to sedate children for dental care. Pediatr Dent 2000; 22(2):107-12.

16. Feld LH, Negus JB, White PF. Oral midazolam preanesthetic medication in pediatric outpatients. Anesthesiology 1990; 73(5):831-4.

17. Fukuta O, Braham RL, Yanase H, Kurosu K. Intranasal administration of midazolam: pharmacokinetic and pharmacodynamic properties and sedative potential. ASDC J Dent Child 1997; 64(2):89-98.

18. LahatE, Goldman M, Barr J, Bistritzer T, Berkovitch M. Comparison of intranasal midazolam with intravenous diazepam for treating febrile seizures in children: prospective randomised study. BMJ 2000; 321(7253):83-6.

19. Aydintug YS, Okcu KM, Guner Y, Gunaydin Y, Sencimen M. Evaluation of oral or rectal midazolam as conscious sedation for pediatric patients in oral surgery. Mil Med 2004; 169(4):270-3.

20. Armijo JA, Herranz JL, Pena Pardo MA, Adin J. Midazolam intranasal y bucal en el tratamiento de las convulsiones agudas. Rev Neurol 2004; 38(5):458-68.

21. Malamed SF. Sedación guía práctica. 3a ed. España: Mosby; 1996.

22. Fukuta O, Braham RL, Yanase H, Kurosu K. The sedative effects of intranasal midazolam administration in the dental treatment of patients with mental disabilities. Part 2: optimal concentration of intranasal midazolam. J Clin Pediatr Dent 1994; 18(4):259-65.

23. Henry RJ, Ruano N, Casto D, Wolf RH. A pharmacokinetic study of midazolam in dogs: nasal drop vs. atomizer administration. Pediatr Dent 1998;20(5):321-326. 